\title{
A comprehensive survey of the current trends and extensions for the proxy mobile IPv6 protocol
}

\begin{abstract}
Network based mobility management has attracted significant research interest due to its salient feature of relieving mobile nodes from participating in the mobility process. This feature of relying the mobility functions on the network entities would indeed eases the deployment of mobility solutions. Proxy Mobile IPv6 (PMIPv6) is considered as a promising network-based mobility management protocol in the next-generation mobile network. However, since the emergence of basic specification of the PMIPv6 protocol, it is still being developed in different directions to enhance its performance in order to ensure the best service for mobile users. This paper presents the PMIPv6 basic specifications and surveys the different extensions that have been considered by both the standardization bodies and researchers to enhance the basic PMIPv6 protocol with interesting features needed to offer a richer mobility experience, namely, clustering, fast handoff, route optimization, network mobility support, and load sharing. The research works conducted for these extensions are analyzed to specify the main issues that should be considered during the design of such extensions. Also, an integrated solution is proposed to show the possibility of combining more than one enhancement feature into a single integrated scheme.
\end{abstract}

Keyword: Clustered PMIPv6; Fast handoff; Load sharing; Network mobility; PMIPv6; Route optimization 